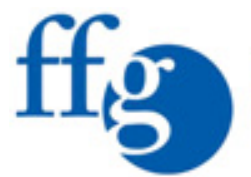

forum für ganzheitsmedizin lorum pour wne medecine intégrale forum per la medizina integrale

Bereits am Ende der 70er-Jahre des letzten Jahrhunderts begann sich die Situation im Gesundheitswesen der Schweiz durch die stetig wachsenden diagnostischen wie therapeutischen Möglichkeiten und die dadurch stark steigenden Kosten spürbar anzuspannen. Die Probleme liessen sich mit dem veralteten Kranken- und Unfallversicherungsgesetz (KUVG) von 1911 immer weniger lösen und wurden vom Parlament mit dringlichen Bundesbeschlüssen oft nur auf später verschoben. Dies führte zu Reglementierungen des Gesundheitswesens und zur Entsolidarisierung in der Krankenversicherung, sodass bald viele Parteien ein Obligatorium verlangten.

Die zunehmende Verstaatlichung sowie die dazugehörende Gefährdung der freien Therapiewahl und Methodenvielfalt wurde uns 1986 erstmals bei einer Revision der Interkantonalen Vereinbarung über die Kontrolle der Heilmittel bewusst. Mit einem umfangreichen Rechtsgutachten von Rudolf Hädener wandten sich deshalb die folgenden fünf Verbände Mitte 1987 erstmals gemeinsam an die zuständigen Behörden: «Vereinigung anthroposophisch orientierter Ärzte in der Schweiz», «Schweizerische Ärztegesellschaft für Erfahrungsmedizin», «Volksgesundheit Schweiz» (heute «vitaswiss»), «Schweizer Kneippverband» und «Verein für ein anthroposophisch erweitertes Heilwesen» (heute «anthrosana»). Diese Ärzte- und Gesundheitsorganisationen mit rund 60000 Mitgliedern bildeten also den Keim des ersten Netzwerks der Komplementärmedizin in der Schweiz.

Im selben Jahr zeigte sich bei der geplanten Teilrevision der Kran-

\title{
Erfolgreiches politisches Netzwerk der Komplementärmedizin
}

In Ergänzung des in dieser Zeitschrift im Oktober 2009 publizierten Beitrags von Dr. med. Hansueli Albonico zur «Komplementärmedizin in der Bundesverfassung der Schweiz» [1] wird hier - ausdrücklich ohne Anspruch auf Vollständigkeit und Objektivität - die Entstehung und wechselvolle Geschichte des «ffg - forum für ganzheitsmedizin» in groben Zügen nachgezeichnet. Der Autor hat 1987 die ursprüngliche Organisation mitbegründet, anfänglich als Geschäftsführer und anschliessend bis 2001 als Co-Präsident (mit-)geleitet. Als Geschäftsführer und Vertreter der Patienten- und Versichertenorganisation «anthrosana» war er bis zur Überführung des «ffg» in den «Dachverband Komplementärmedizin» im Oktober 2009 immer mit viel Herzblut aktiv mit dabei.

ken- und Mutterschaftsversicherung (KMVG) eine ähnliche Problematik. $\mathrm{Zu}$ einem Paket geschnürt wollten Bundesrat und Parlament eine Mutterschaftsversicherung sowie dazu auch gleich noch den (schul-)wissenschaftlichen Nachweis für Untersuchungen, Behandlungsmethoden und Heilmittel in der Krankenversicherung verankern. Auf Anstoss eines initiativen Mitbürgers und Lehrers an der Plastikschule in Dornach, Ueli Hintermann, konnten wir rasch mit professioneller und kostenloser Hilfe einer bekannten politischen Agentur in Zürich in allen grösseren Tageszeitungen insgesamt rund 60 Inserate mit dem Titel «Mutterschaftsschutz mit Kuckucksei» schalten. Damit sind wir erstmals vor der Volksabstimmung vom 6. Dezember 1987 mit dem Namen «Unabhängige Gemeinschaft für Freiheit im Gesundheitswesen» (UGFG) öffentlich gegen die Einschränkung der freien Therapiewahl aufgetreten, und dies erfolgreich, denn die KMVG-Teilrevision wurde abgelehnt.

Die Gefahr war jedoch keineswegs gebannt. Gleich anschliessend wandten wir uns deshalb mit einer von Dr. med. Peter Heusser verfassten
Stellungnahme an den zuständigen Bundesrat Flavio Cotti und das Parlament. Glücklicherweise erhielten wir dabei unerwartete und dann während sieben Jahren äusserst wertvolle Unterstützung durch Rudolf Hafner, der 1984 als Revisor der kantonalen Finanzkontrolle mit seinen Enthüllungen zum Finanzgebaren der Berner Regierung ein politisches Erdbeben ausgelöst hatte. Auf Anhieb war er in den Grossrat und bei den Parlamentswahlen Ende 1987 in den Nationalrat gewählt worden. Von Beginn seiner Tätigkeit im Bundesparlament weg engagierte sich Rudolf Hafner mit Vorstössen und Interventionen bei Behörden für die Freiheit im Gesundheitswesen und die Anliegen der Komplementärmedizin. Durch seine intensiven Bemühungen beim Bundesamt für Sozialversicherung konnten unter anderem erstmals von uns vorgeschlagene Vertreter in wichtigen Kommissionen Einsitz nehmen. So wurden 1990 die Apothekerin Silvia Briggen - anstelle eines FMH-Vertreters! - in die Arzneimittel- und Dr. med. Andreas Beck in die Leistungskommission gewählt.

Erfreulicherweise konnten wir Nationalrat Hafner nach einiger Zeit als

\section{KARGER}

Fax +49761 4520714

E-Mail Information@Karger.de

www.karger.com
(๑) 2010 S. Karger GmbH, Freiburg

www.karger.com/szg
Herbert Holliger

anthrosana - Verein für anthroposophisch erweitertes Heilwesen

Postplatz 5, Postfach 128, 4144 Arlesheim, Schweiz

Tel. +41 61 70115-14, Fax -03

info@anthrosana.ch 
Delegierten für Öffentlichkeitsarbeit unseres Dachverbandes gewinnen und für sein Engagement entschädigen. Die inzwischen um drei Ärztegesellschaften gewachsene, aber rechtlich weiterhin als einfache Gesellschaft funktionierende «Unabhänge Gemeinschaft» gab sich der einfacheren Kommunikation zuliebe, aber auch um den offenen und demokratischen Forumscharakter zu betonen, im Frühjahr 1991 einen kürzeren Namen: «Forum Freiheit im Gesundheitswesen» (FFG). Die sich regelmässig etwa fünfmal jährlich in Olten treffenden Gesellschafter konnten wachsendes Vertrauen zueinander gewinnen und politische Kompetenz erwerben, da fast immer ein äusserst erfahrener Vertreter der bereits genannten Agentur ohne Honorar, aber mit viel Hintergrundwissen teilnahm. Dadurch war es dem FFG möglich, professionelle Medienkonferenzen zu gesundheitspolitischen Themen oder Volksabstimmungen zu organisieren und sich bei Medienvertretern sowie Behörden zunehmend Gehör zu verschaffen.

Fünf Jahre nach der Gründung wurden auf verschiedenen Ebenen Aktivitäten mit zum Teil äusserst nachhaltiger Wirkung für die Komplementärmedizin unternommen. Auf Empfehlung von Nationalrat Hafner konnte das FFG Anfang 1992 bei Prof. Peter Saladin ein Gutachten zu Rechtsschutzproblemen im Zusammenhang mit dem Heilmittelkonkordat in Auftrag geben. Kurz nach der Publikation und dringenden Empfehlung, der neuen Interkantonalen Vereinbarung (IKV) nicht beizutreten, lehnte der Zürcher Kantonsrat dies tatsächlich ab. Damit war die bereits von vielen Kantonen unterzeichnete IKV plötzlich hinfällig und die Ausgangslage für eine Bundeslösung bei der Heilmittelkontrolle, die schlussendlich zum Schweizerischen Heilmittelinstitut «Swissmedic» geführt hat, geschaffen.

Im gleichen Jahr wurde die Krankenkassen-Initiative auch vom FFG erfolgreich bekämpft und die von Rudolf Hafner lancierte kantonale Volksinitiative für einen Lehrstuhl für Naturheilverfahren an der Universität Bern unterstützt, welche 1996 zur KIKOM führte.

Aufgrund der kaum noch zu lösenden Probleme im Gesundheitswesen hatte der Innenminister Flavio Cotti bereits 1991 einer von Ständerat Schoch geleiteten Parlamentariergruppe den Auftrag erteilt, eine Totalrevision des KUVG mit einer obligatorischen Krankenversicherung vorzubereiten. Von Beginn weg versuchte das FFG mit Gesprächen, Vorstössen und Stellungnahmen zu verhindern, dass die Komplementärmedizin über den (schul-)wissenschaftlichen Nachweis der Wirksamkeit ganz an den Rand oder in die nicht allen zugänglichen Zusatzversicherungen gedrängt wird. Trotz konkreter Versprechen sogar von Bundesrätin Ruth Dreifuss, welche 1993 das Departement von Flavio Cotti und damit das geplante neue KVG übernommen hatte, drohte das FFG mit dem Referendum und brachte dieses 1994 mit 58000 Unterschriften problemlos zustande.

Im Hinblick auf die Volksabstimmung vom 4. Dezember 1994 folgte eine intensive Kampagne mit Flugblättern und Inseraten, Medienkonferenz und der Teilnahme an vielen Podien wie auch zweimal mit einer Delegation in der damals sehr populären, von Filippo Leutenegger geleiteten ARENA am Schweizer Fernsehen. Auf diese einmalige und vom FFG geschickt genutzte Chance zur Bekanntmachung und Durchsetzung der bedrohten Anliegen der Komplementärmedizin gehen die von Bundesrätin Dreifuss dann tatsächlich umgesetzten Versprechen zu ihrer Berücksichtigung zurück. Zu den vielen, inzwischen in der Spezialitätenliste enthaltenen Heilmitteln wurden 1999 - drei Jahre nach Inkraftsetzung des neuen KVG - auch die ärztlichen Leistungen von fünf komplementärmedizinischen Richtungen provisorisch in die Grundversicherung aufgenommen. Die zum Teil dramatischen Vorgänge um die mit sechs Millionen Franken dotierte PEK-Sudie zur Evaluation dieser Methoden bis 2005 wurden hier von Dr. med. Hansueli Albonico ausführlich beschrieben [1].

Inzwischen hatte sich das FFG unter dem langjährigen Co-Präsidium von Dr. med. Ulrich Heusser-Buchs und mir durch weitere Verbände verstärken können. So war 1994 der neu gegründete Schweizerische Verband für komplementärmedizinische Heilmittel (SVKH) mit seiner in Heilmittelfragen sehr kompetenten Geschäftsführerin, Sabine Hockenjos, dazugestossen. 1995 schlossen sich dann auch die Ärztegesellschaften in der UNION schweizerischer komplementärmedizinischer Ärzteorganisationen zusammen. Diese Professionalisierung verschaffte dem FFG wesentlich mehr fachspezifische Kompetenz und - durch die allmählich ebenfalls dazugekommenen Vertreter komplementärmedizinischer Kliniken und Spitäler sowie einzelner Therapeutenverbände - eine noch breitere Abstützung und grössere Glaubwürdigkeit.

Um die inzwischen ganz auf die Komplementärmedizin fokussierte Zielsetzung zum Ausdruck zu bringen, nannte sich die Dachorganisation ab 1999 «ffg - forum für ganzheitsmedizin». 2002 wurde diese rechtlich in einen Verein mit einem aus sieben Mitgliedern bestehenden Vorstand übergeführt. In den Statuten wurde speziell darauf geachtet, dass schnelle Aktionen - wie die erfolgreiche Petition zum Erhalt der Heilmittelvielfalt vom Juni 2001 - durchgeführt werden können. Ausserdem wollte das ffg neu auch nichtärztlichen Heilpraktikerund Therapeutenverbänden offenstehen. Bereits nach zwei Jahren trat jedoch der ganze Vorstand zurück und dem ffg drohte die baldige Auflösung.

Zur Abwendung des voraussehbaren PEK-Debakels mit dem seit 2003 zuständigen Bundesrat Pascal 
Couchepin konnten wir jedoch inzwischen in einer kleinen Arbeitsgruppe seit langem engagierter Persönlichkeiten unter der Leitung von Dr. HansPeter Studer die Lancierung einer Volksinitiative vorbereiten und auf der Suche nach einem geeigneten Rechtsträger damit sogar das ffg $\mathrm{zu}$ neuem Leben erwecken. Ein kleiner Vorstand mit der Präsidentin Birgitt Kuster und dem Kassier Christoph Oling sowie Herbert Schwabl und später Willy Bernhard übertrugen im Namen des Vereins die Arbeiten über ein Projektreglement an den vom ffg gewählten Lenkungsausschuss und die Geschäftsstelle von Elisabeth Sulger Büel. Diese kümmerten sich allen Widerständen zum Trotz erfolg- reich um die Organisation und Finanzierung der Unterschriftensammlung, Lobbyarbeit im Parlament und - nach dem sinnvollen Rückzug der im September 2005 mit fast 140000 Unterschriften eingereichten Volksinitiative - um die Kampagne für die Volksabstimmung zum Verfassungsartikel «Zukunft mit Komplementärmedizin» vom 17. Mai 2009.

Die mit $67 \%$ der Stimmenden deutliche, in sämtlichen Kantonen erreichte Zustimmung führte zur weltweit erstmaligen Verankerung der Komplementärmedizin in einer Staatsverfassung, welche auch international grosse Anerkennung fand. Damit hat das ffg nach kontinuierlicher Aufbauarbeit während 22 Jahren und vielen überwundenen Schwierigkeiten einen kaum je erhofften politischen Erfolg errungen. Mit der Überführung in den «Dachverband Komplementärmedizin» lebt das 1987 gegründete Netzwerk mit veränderten Strukturen sowie neuem Namen weiter und wird sich hoffentlich ebenso erfolgreich um die nun dringende Umsetzung des Verfassungsartikels kümmern können.

\section{Literatur}

1 Albonico $\mathrm{H}$ : Komplementärmedizin in der Bundesverfassung der Schweiz: «Fallstudie» zur Verschränkung von Politik und Wissenschaft. Schweiz Zschr Ganzheitsmedizin 2009;21:294-299. 